X.B. Yang

G.D. Xu

http://dx.doi.org/10.21278/brod69406

ISSN 0007-215X

eISSN 1845-5859

\title{
NUMERICAL SIMULATION OF THE OBLIQUE WATER ENTRY OF WEDGES WITH VORTEX SHEDDING
}

UDC 629.5.016.8

Original scientific paper

\begin{abstract}
Summary
The hydrodynamic problem of the water entry of wedges with oblique velocity has been investigated numerically. The simulations of the interaction of the wedge and initial calm surface are carried out by solving the Reynolds-averaged Navier-Stokes equations (RANS). The overset mesh technique is introduced and the air-water interface is tracked using the method of Volume of Fluid (VOF). The water entries of wedges with various deadrise angles at different oblique velocities are simulated. A noticeable vortex flow and low pressure area on the leeward side of the wedge have been observed. The evolution of vortex and the pressure distribution over the wedge surface are analysed. The local vortex flow affects the local hydrodynamic pressure significantly.
\end{abstract}

Key words: $\quad$ Numerical simulation; oblique water entry; vortex shedding; overset mesh technique;

\section{Introduction}

The hydrodynamic impact, including the slamming of ships with V-shaped bow or planning vessels, the ditching of seaplane, would cause the damage of structures. The impact usually last a short period and the problem can be treated as the water entry of wedges. The Mach number of the fluid flow during impact of these marine structures is usually negligible, and the compressibility of the fluid can be ignored (Newman [1]). The analytical solution was firstly proposed by Von Karman [2] based on momentum conservation. Wagner [3] took account the linear free surface effect; the modification was effective in predicting the overall impact loads at initial stage. The matched asymptotic method was further developed to by Armand and Cointe [4], Korobkin and Puknachov [5], Korobkin [6], Howison, Ockendon and Oliver [7], and the water jet was included. Mei, Liu and Yue [8] developed an analytic solution based on the generalized Wagner theory through boundary-integral equation method; the conformal mapping technique was adopted.

Efforts have been made to simulate the water entry using nonlinear free surface theory. For wedge water entry problem at constant velocity, the time and spatial variables can be combined and the flow becomes self-similar when the gravity effect and the viscosity have 
been ignored. The nonlinear similarity solution of vertical water entry of symmetric wedge had been obtained by Dobrovolskaya [9] through conformal mapping. Zhao and Faltinsen [10], Battistin and Iafrati [11] studied the vertical water entry of wedges through time stepping schemes, where the nonlinear free surface boundary conditions had been satisfied. $\mathrm{Wu}$, Sun and $\mathrm{He}$ [12] and $\mathrm{Xu}$, Duan and $\mathrm{Wu}$ [13] further investigated the free fall motion of wedges; the auxiliary function had been introduced to decouple the interactive motions of the fluid and structures. Except inviscid theory, numerical simulations of water entry problems were carried out through solving Navier-Stokes (N-S) Equations. Fairlie-Clarke and Tveitnes [14] simulated the water entry of wedges using the finite volume method based on commercial computational fluid dynamics (CFD) code Fluent. Shademani and Ghadimi [15] introduced a two dimensional two-phase Finite-Element method (FEM) to study the hydrodynamic impact force of a wedge, and the cavity and the secondary impact of the returning free surface had been investigated.

It happens that the wedge-shaped structure impacts water surface with inclined angle and/or oblique velocity. Xu, Troesch and Vorus [16] studied the impact of asymmetric planning vessel. Garabedian [17] and Chekin [18] had developed similarity solution for the asymmetric water entry problem. Semenov and Iafrati [19] analysed the similarity solution of asymmetric wedge water entry through conformal mapping of complex flow. $\mathrm{Xu}$, Duan and $\mathrm{Wu}$ [20] found the similarity solution through iterations based on boundary element method. Negative pressure at the apex on the leeward side of the wedge, which may cause the cavitation-ventilation, had been observed. The typical experimental and theoretical study on the oblique water entry of asymmetric wedge had been carried out by Judge, Troesch and Perlin [21]. Two types of impact, with and without separation-ventilation at the wedge apex, had been investigated. They attempted to address the criteria for the ventilation at the wedge apex when the inclined angle or ratio of horizontal and vertical velocity increases. Agreement of the experiment and the predicted separation-ventilation at the wedge apex had been found. $\mathrm{Gu}$ et al [22] had analysed the oblique water entry of wedges based on two phase flow theory and the level set method. The ventilation during water entry had been analysed. Javanmardi, Ghadimi and Tavakoli [23] studied the water entry of a propeller blade impacting on the water surface, the cavitation and ventilation effects on the hydrodynamic characteristic were investigated through Finite volume method. Except the separation-ventilation during the wedge water entry, vortex shedding would develop on the leeward side of the wedge apex in the case of attached flow impact. Low pressure does not necessarily result the ventilationcavitation since more physical conditions are required. The flow pattern and the pressure distribution require further investigations.

For attached flow, vortex shedding occurs at the sharp edge due to large velocity gradient when there exists transverse flow (Bacthelor [24]). Pullin [25], Graham [26] and Pullin and Perry [27] have investigated the vortex shedding of wedges theoretically and/or experimentally, although there is reasonable discrepancy. For the oblique water entry of a wedge, the vortex shedding is developed due to large gradient of pressure on the leeward side. The vortex flow will affect the flow pattern and pressure distribution significantly in return. Riccardi and Iafrati [28] introduced point vortex to circumvent the singularity at wedge apex. However, the free surface was assumed quiescent. $\mathrm{Xu}$ and $\mathrm{Wu}[29]$ studied the water entry of a wedge at oblique velocity using a time stepping scheme, the vortex shedding is approximated by using point vortex. The Kutta condition was satisfied when solving the boundary integral equation, and the pressure jump at the wedge apex had been avoided. Semenov and Wu [30] considered the water entry of wedges with vortex wake in the framework of potential flow. The vortex flow was obtained through the integral hodograph method combined with Birkhoff-Rott method. Besides the inviscid theory, the hydrodynamic problem can be 
modeling through CFD by solving the N-S equations. The effects of viscosity and the dissipation of the vortex flow can be included.

Present study concerns the oblique water entry of wedges with various deadrise angles. In physics, the breath of a wedge is usually finite. If the mass of whole structure is large, like a seaplane or a lifeboat, the variation of velocity due to local impact would be less significant at the initial stage of the impact. It becomes practical to study the water entry of a finite wedge with constant velocity, which makes the problem simpler. The interactions of the wedge and flow are simulated through solving the Reynolds Averaged Navier-Stokes equations (RANS). The realizable $k-\varepsilon$ model [31] with high $\mathrm{y}+$ wall treatment is adopted. The overset mesh technique is introduced. Volume of fluid (VOF) method [32] is used to capture the interface of water and air. It is observed that the vortices have been developed as the wedge entering initial quiescent water surface. As the vortex rolls up, the low pressure area close to the wedge apex expands. The pressure distributions with various deadrise angles and oblique velocity are analysed.

\section{Numerical methods}

The hydrodynamic problem of the oblique water entry of a wedge is considered. The Cartesian coordinate system $o-x-y-z$ is defined with $x$ axis along the undisturbed water surface and $y$ axis pointing upwards. As shown in Fig.1(a), the breath of the wedge is denoted as $B$, and the deadrise angles is $\beta$. The wedge impacts on the initial calm water surface with prescribed vertical velocity $V$ and horizontal velocity $U$, and we have $\varepsilon=U / V$. A moving coordinate system $o-x^{\prime}-y^{\prime}-z^{\prime}$ which is fixed with the wedge is defined; its origin is located at the apex of the wedge and its horizontal axis and vertical axis are parallel with the axes of the $0-x-y-z$ coordinate system at beginning of the impact. The wedge enters water with constant velocity during the whole process, there is no angular velocity. It moves only in the horizontal direction and vertical direction.

The concerned velocity of the wedge is much lower than the speed of sound, the compressibility of the fluid is therefore ignored. The fluid is treated as incompressible and the density is constant. The conversation equations of mass, momentum and energy are satisfied. The partial differential equation for the mass conservation is expressed as

$$
\frac{\partial u_{i}}{\partial x_{i}}=0
$$

where $i=1,2,3, x_{1}=x, x_{2}=y, x_{3}=z$, and $u_{i}$ is the velocity components.

The conservation of the momentum equation is written as

$$
\frac{\partial u_{i}}{\partial t}+u_{j} \frac{\partial u_{i}}{\partial x_{j}}=-\frac{1}{\rho} \frac{\partial p}{\partial x_{i}}+\frac{\mu}{\rho} \frac{\partial^{2} u_{i}}{\partial x_{j} \partial x_{j}}+\frac{f_{b}}{\rho}
$$

where $\mu$ is the coefficient of viscosity, $\frac{\partial p}{\partial x_{i}}$ is the surface force, $f_{b}$ is the body force acting on the fluid volume and $f_{b}=g$.

The VOF is used to track the interface of the two phase flow, we have

$$
\frac{\partial}{\partial t}(\alpha)+\nabla \cdot(\alpha \vec{V})=0
$$


where $\alpha$ is the volume fraction of the water phase in each cell. If $\alpha=0$, the cell will be filled with air; the cell is filled with water when $\alpha=1$; if $0<\alpha<1$ the cell will be filled with both water and air as the interface of the two phases.

\section{Convergence study and validation}

The numerical simulations of the water entry of wedges are conducted using commercial CFD code STAR-CCM+(version 11.06). The interactions of the fluid and wedges are simulated by solving the RANS equations through Finite Volume Method (FVM). The VOF is adopted to capture the free surface flow. The turbulent flow is modelled by the realizable $k-\varepsilon$ model. The overset mesh method is introduced. There are two sets of meshes, one is the overset mesh block moving with the wedge, and the other is the background mesh which contains the regions of air and water. The interpolation boundary exchanges data between the region of overset mesh block and the region of background, as shown in Fig. 1(b). The near wall prism layer is employed on the wedge surface in the overset region, as shown in Fig. 1(c). A local mesh refinement is conducted, and large meshes far away from the wedge are used. The wedge water entry is treated as a two-dimensional problem; the background fluid domain contains only two mesh layers in the direction of $z$ axis. The requirements of memory and CPU have been reduced significantly.

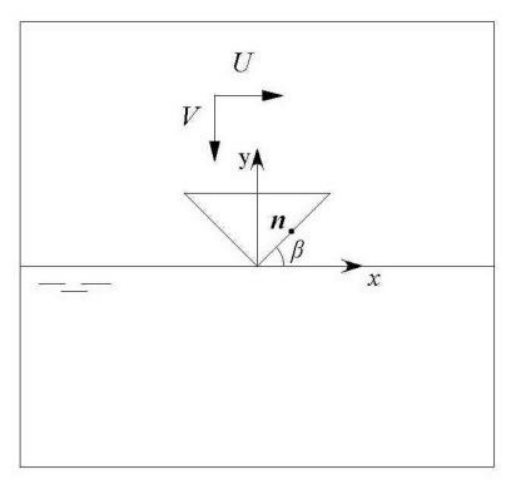

(a)

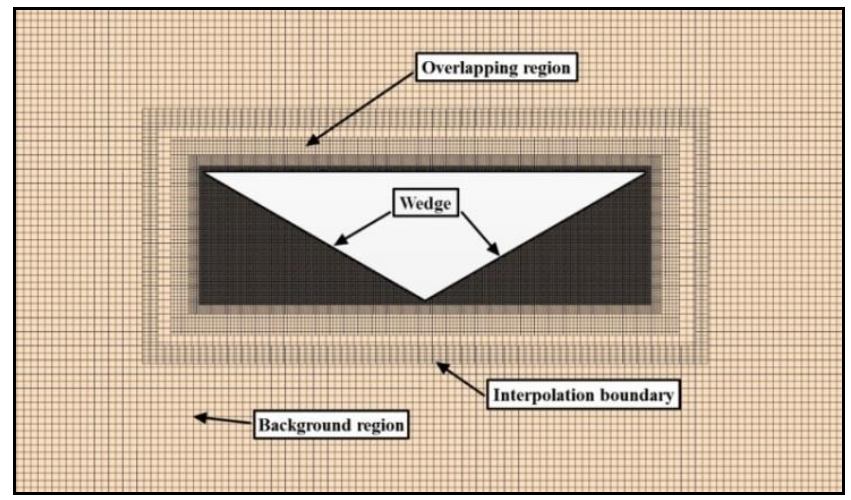

(b)

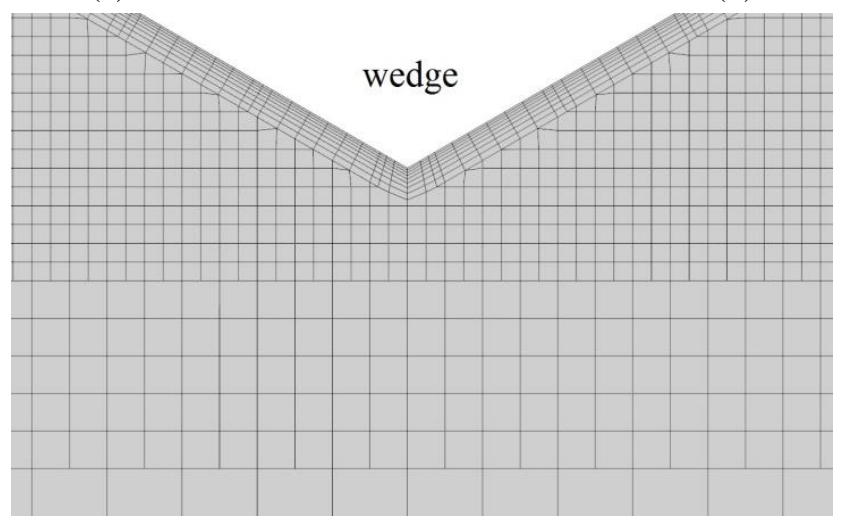

(c)

Fig.1 (a) The sketch of the wedge water-entry problem, (b) the overset mesh and (c) refined mesh near the wedge surface

The convergence study on mesh size and time resolution is carried out firstly. The vertical water entry of a symmetric wedge with deadrise angle $\beta=45^{\circ}$ is simulated. The breath of the wedge $B=0.05 \mathrm{~m}$. The vertical velocity $V=5 \mathrm{~m} / \mathrm{s}$. Two sets of meshes and two different time step intervals for each set of meshes have been used. The parameters of fundamental mesh size $\Delta x$ near the wall of wedge and time resolution $\Delta t$ are listed in table 1. The thickness of near wall prism layer is $1.0 \times 10^{-5} \mathrm{~m}$ and there are 10 mesh layers parallel the wall of the wedge. As shown in Fig.2, the pressure distributions on the wedge surface are 
in good agreement except case 1. The pressure distribution and the vertical force are converged when mesh size $\Delta x<1.0 \times 10^{-4} \mathrm{~m}$ and time resolution $\Delta t<1.0 \times 10^{-5} \mathrm{~s}$. Comparing the pressure distribution with the similarity solution of $\mathrm{Xu}$, Duan and $\mathrm{Wu}$ [20], good agreement has been found, as shown in Fig.2(b). In table 1, the vertical impact forces at the depth $s=V t=0.6 B$ are in good consistence. We note that the results of $\mathrm{Xu}$, Duan and $\mathrm{Wu}[20]$ ignored the effects of viscosity and the gravity. Present numerical results indicate that the effects of gravity and viscosity are less significant during the initial stage of the impact.

Table 1 The mesh size and time resolution of the simulations, and the corresponding vertical force at $V t / B=0.6$

\begin{tabular}{|c|c|c|c|c|}
\hline Case & 1 & 2 & 3 & 4 \\
\hline Grids number & 1618324 & 1618324 & 2551573 & 2551573 \\
\hline$\Delta t(s)$ & $2.0 \times 10^{-5}$ & $1.0 \times 10^{-5}$ & $1.0 \times 10^{-5}$ & $5.0 \times 10^{-6}$ \\
\hline$\Delta x(m)$ & $1.0 \times 10^{-4}$ & $1.0 \times 10^{-4}$ & $5.0 \times 10^{-5}$ & $5.0 \times 10^{-5}$ \\
\hline $\mathrm{F}_{\mathrm{y}} / 0.5 \rho V^{2}(V t)$ & 6.943 & 6.975 & 6.962 & 6.971 \\
\hline
\end{tabular}

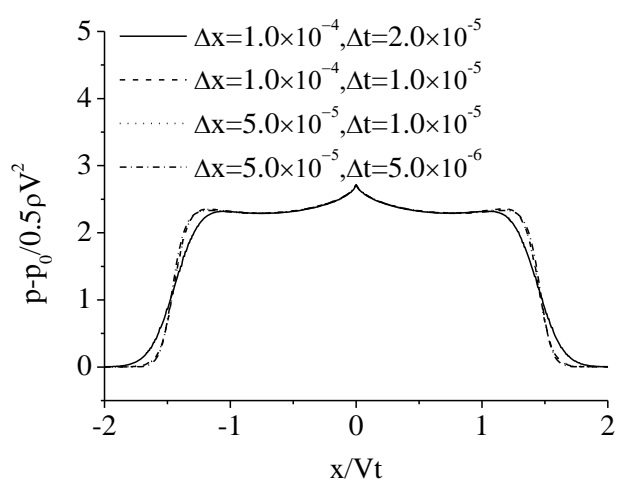

(a)

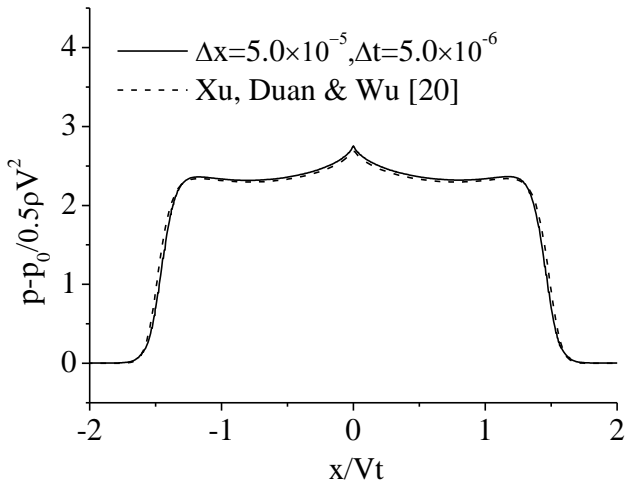

(b)

Fig.2 Pressure distribution of vertical water entry of a wedge with $\beta=45^{\circ}, V t / B=0.6$, (a) results of different meshes and time resolutions, (b) comparison with similarity solution.

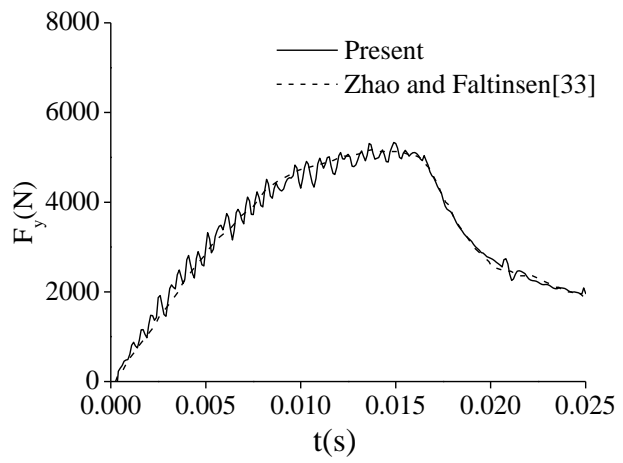

(a)

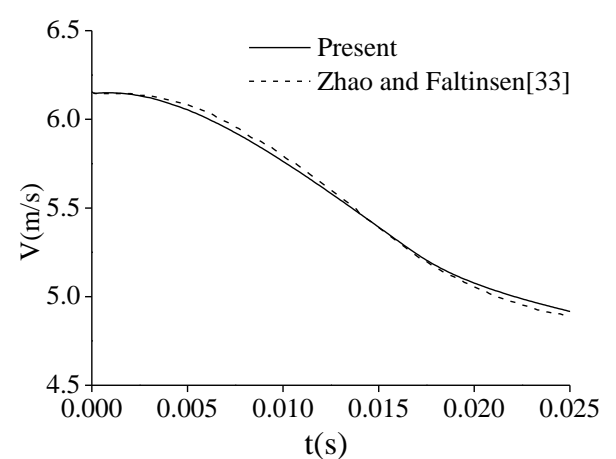

(b)

Fig.3 (a) The hydrodynamic force on the wedge, (b) velocity.

The free fall water entry of a wedge with $\beta=30^{\circ}$ is simulated. The wedge is 1.0 meter in length and $0.5 \mathrm{~m}$ in breath, the mass $m=255.5 \mathrm{~kg}$. The vertical velocity $V=6.15 \mathrm{~m} / \mathrm{s}$. The results are validated through comparing with the experimental data of Zhao and Faltinsen [33]. The length of the measured section is $0.2 \mathrm{~m}$. As shown in Fig.3, the vertical force on the 
measured section increases as the wedge entering water; it decreases after the wedge is below the initial calm waterline. The vertical force and velocity agree well with the experimental results.

Table 2 The typical characteristics of mesh size and time resolution in the simulations with $\beta=45^{\circ}$ and

\begin{tabular}{|c|c|c|c|c|c|}
\hline \multicolumn{7}{|c|}{$\varepsilon=0.3}$. \\
\hline Case & 1 & 2 & 3 & 4 & 5 \\
\hline$\Delta x(m)$ & $1.0 \times 10^{-4}$ & $1.0 \times 10^{-4}$ & $5.0 \times 10^{-5}$ & $5.0 \times 10^{-5}$ & $2.5 \times 10^{-5}$ \\
\hline$\Delta t(s)$ & $2.0 \times 10^{-5}$ & $1.0 \times 10^{-5}$ & $1.0 \times 10^{-5}$ & $5.0 \times 10^{-6}$ & $5.0 \times 10^{-6}$ \\
\hline
\end{tabular}

We further study the independence of the mesh size and the time resolution in the case of oblique water entry. The vertical velocity $V=5 \mathrm{~m} / \mathrm{s}$ and $\varepsilon=U / V=0.3$ are prescribed. The typical mesh sizes and time resolutions are shown in table 2. Simulations with three sets of meshes and three different time resolutions are conducted. The horizontal and vertical forces against time are shown in Figs. 4(a) and 4(b) respectively. The forces increase linearly at the beginning of the impact and they drop when the flow separates at the knuckles. In Fig. 5(a) the free surface profile at $V t / B=0.6$ is presented. As shown in Fig. 5(b), the pressure distributions found their agreement when different mesh sizes and time resolutions are adopted. The numerical results are converged. The velocity field of case 3 at $V t / B=0.72$ are further shown in Fig.6. A stagnation point on the windward side (right wedge surface) can be observed. The vortex develops on the leeward side (left wedge surface). Another stagnation above the vortex core on the leeward side is found. A higher pressure at these stagnations can be found. The pressure close to the vortex core drops significantly. This low pressure area will further expand as the evolution of the vortex. This will be further studied in the following section. The typical mesh size and time resolution of case 3 in table 2 will be adopted.

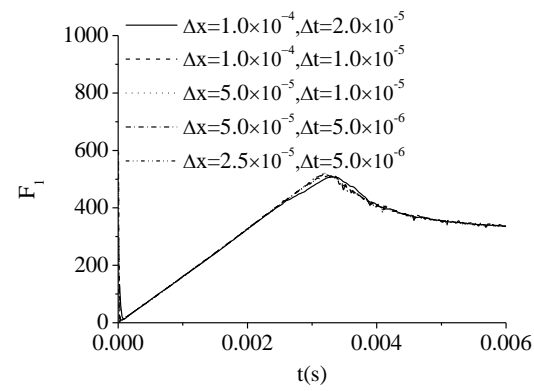

(a)

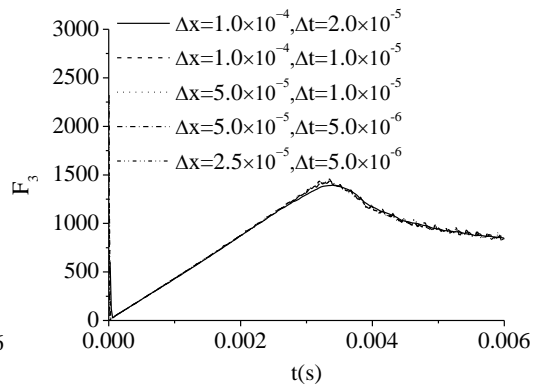

(b)

Fig.4 The time history of (a) horizontal force and $F_{1}$, and (b) vertical force $F_{3}$.

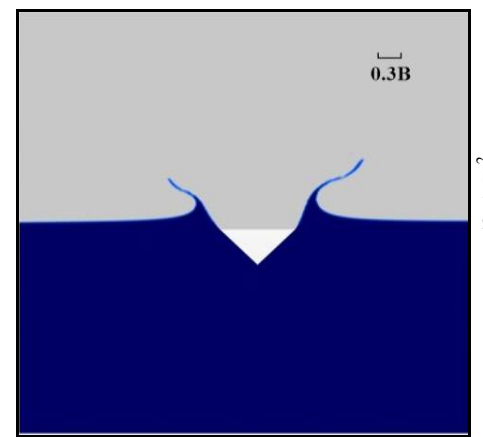

(a)

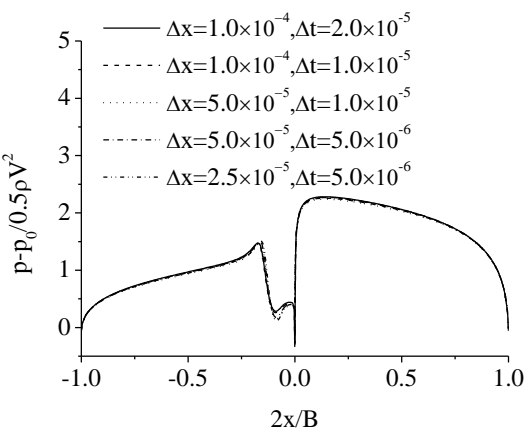

(b)

Fig.5 water entry of wedge with deadrise angle $\beta=45^{\circ}$, (a) The free surface profile and (b) pressure distribution at $V t / B=0.6$. 


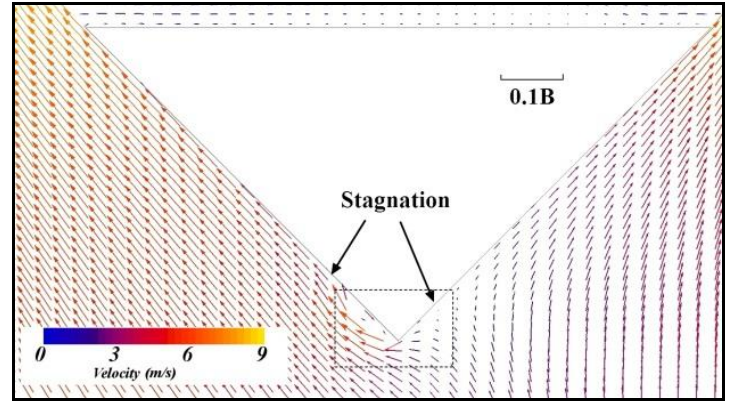

(a)

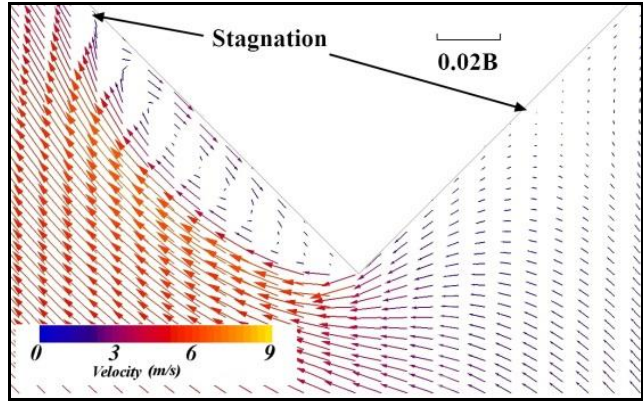

(b)

Fig.6 (a) The velocity field and (b) enlargement of the local flow field near the wedge apex when $V t / B=0.6$, the deadrise angle wedge $\beta=45^{\circ}$.

The oblique water entry of wedge is further validated. In the work of Russo et al [34], the wedge's deadrise angle $\beta=37^{\circ}, v=\sqrt{U^{2}+V^{2}}=2.75 \mathrm{~m} / \mathrm{s}$ and $\varepsilon=0.364$. The mass of the wedge is $0.89 \mathrm{~kg}$, the length is $0.19 \mathrm{~m}$ and the width is $0.2 \mathrm{~m}$. The wedge slides along an oblique bar and impact on the water surface. A pressure sensor (PS) is placed on the right hand side of wedge surface at point $n$, which has a distance $0.078 m$ from the wedge apex. We define the oblique displacement $r=\sqrt{q^{2}+s^{2}}$, where $q$ is transverse displacement, and $s$ is the vertical displacement. The numerical results are compared with the experimental results of Russo et al [34]. As shown in Fig.7, the oblique displacement, velocity and acceleration are in good agreement. Fig.7d shows the time history of the pressure at point $n$. The calculated pressure and the pressure obtained from pressure sensor and the pressure reconstructed from the PIV velocity field agree well with each other. The difference between numerical results and experiments data are mainly due to the friction of experimental apparatus and the resulting discrepancy of velocity.

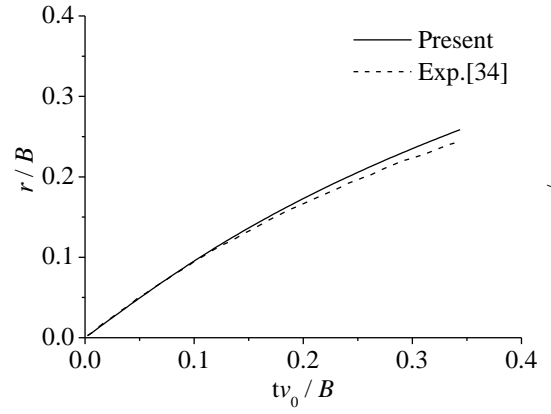

(a)

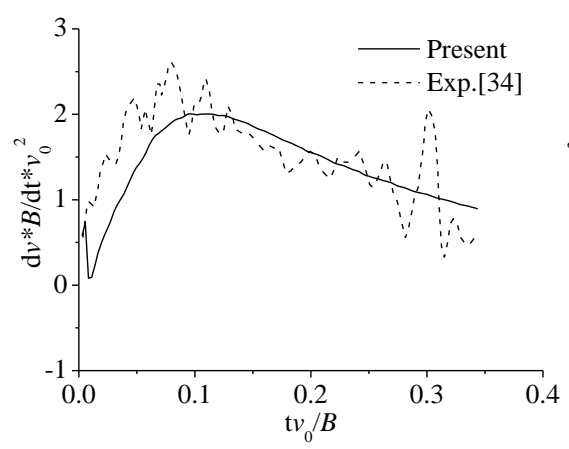

(c)

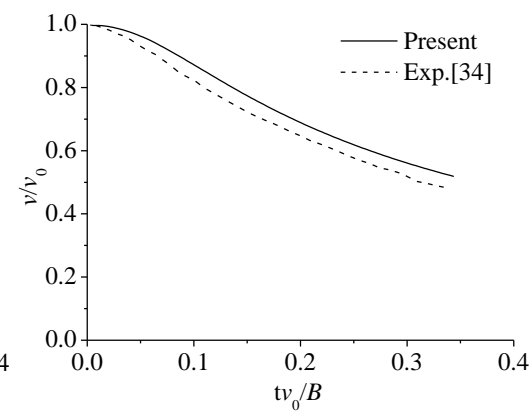

(b)

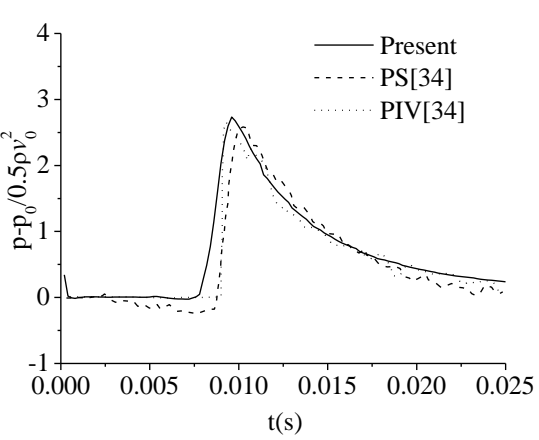

(d)

Fig.7 Results of oblique water entry of a wedge with $\beta=37^{\circ}$ and $\varepsilon=0.364$, (a) displacement, (b) oblique velocity, (c) oblique acceleration, (d) time history of pressure at point $n$. 


\section{Numerical results and discussions}

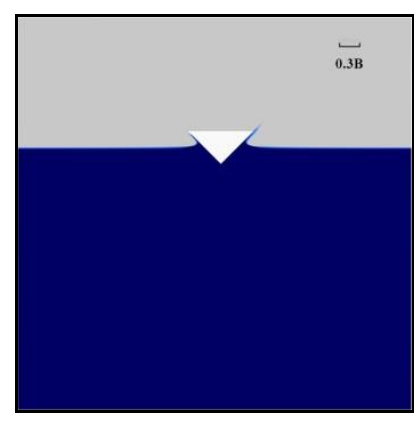

(a)

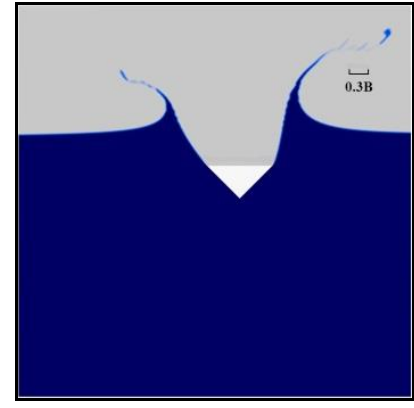

(d)

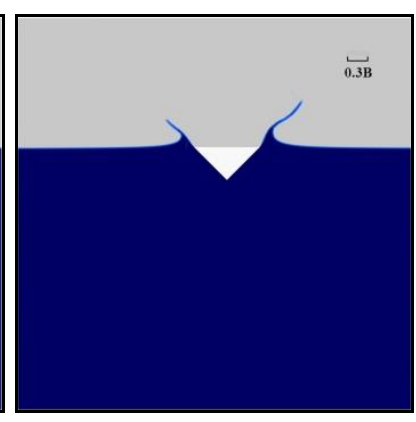

(b)

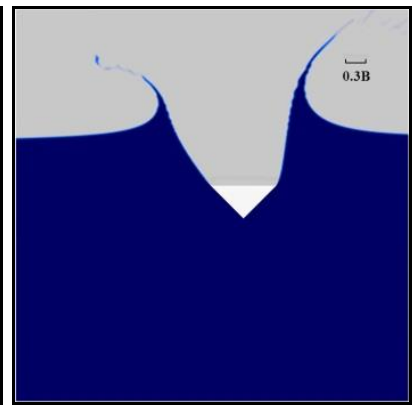

(e)

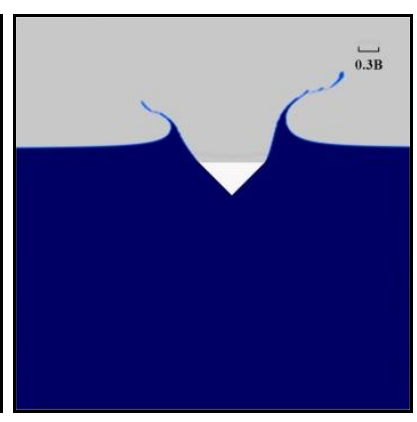

(c)

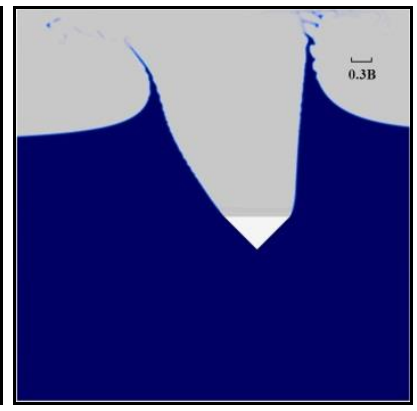

(f)

Fig. 8 Free surface elevation during the water entry of a wedge with $\beta=45^{\circ}, \varepsilon=0.4$ at (a) $V t / B=0.24$, (b) $V t / B=0.48$, (c) $V t / B=0.72$, (d) $V t / B=0.96$ (e) $V t / B=1.20$ (f) $V t / B=1.68$.

The oblique water entry of a wedge with small to medium $\varepsilon=U / V$ would not necessarily result flow detachment-ventilation at the wedge apex. In the case of attached flow water entry, the vortex will shed from the wedge apex and roll up due to the transverse flow. This phenomenon has been omitted in the experiment. The water entry of a finite wedge with deadrise angle $\beta=45^{\circ}$ and breath $B=0.05 \mathrm{~m}$ has been simulated. The entry speed $V=5 \mathrm{~m} / \mathrm{s}$ and $\varepsilon=0.4$. The free surface profile at various entry distances are shown in Fig.8. The water piles up and the jet flow moves along the wedge surface in initial stage. The flow detachment at the wedge knuckles occurs and a large cavity forms as the wedge entering water further. Fig.9 presents the pressure contour and the local flow velocity at $V t / B=0.24, V t / B=0.48$, $V t / B=0.72, V t / B=0.96, V t / B=1.20$ and $V t / B=1.68$. As shown in Fig. 8(a), the highest pressure at $V t / B=0.24$ appears at the jet root on the windward side and the low pressure zone is found close to the apex on the leeward side of the wedge. After the jet flow separates at the knuckles, the high pressure area moves from the jet root to the zone approaching the wedge apex. The transverse flow at the wedge apex induces the vortex flow. The vortex evolves and expands. The low pressure area on the leeward side expands as the vortex develops, see Fig.9. It develops into an elliptic-shaped vortex bolt which elongated along the leeward side of the wedge surface. Similar to Fig.6, the stagnations of the velocity on the two sides of the apex can be observed. Fig.10 shows the pressure distribution on the wedge surface at various entry distances. The pressure is higher at the initial stage of the water entry. Comparing with similarity solution of $\mathrm{Xu}$, Duan and $\mathrm{Wu}[20]$, most part of pressure distribution at $V t / B=0.24$ agree each other, but discrepancy near the wedge apex can be observed, as shown in Fig.10(b). The low pressure area is larger than that of the inviscid theory. After the jet flow detaches the knuckles, the pressure at the knuckle decreases significantly and equals the ambient air pressure. The peak pressure moves to the wedge apex where the flow stagnation can be found. The lowest pressure due to vortex flow is found on the leeward side of the wedge. The low pressure area expands as the development of vortex. The highest pressure on the leeward side, which is corresponding to the stagnation point, is moving away 
from the apex as the wedge entering water. We further compare the pressure distribution obtained from the inviscid theory by $\mathrm{Bao}, \mathrm{Wu}$ and $\mathrm{Xu}$ [35], the discrepancy caused by the vortex flow on the leeward side are significant at $V t / B=0.72$ and $V t / B=1.20$, as shown in Figs.10(c) and 10(d). The pressure distribution in Fig.10 indicates that vortex flow affects the local pressure significantly during oblique water entry.

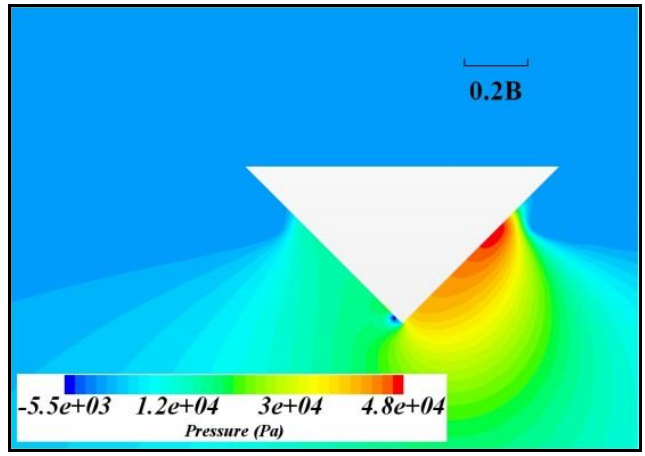

(a)

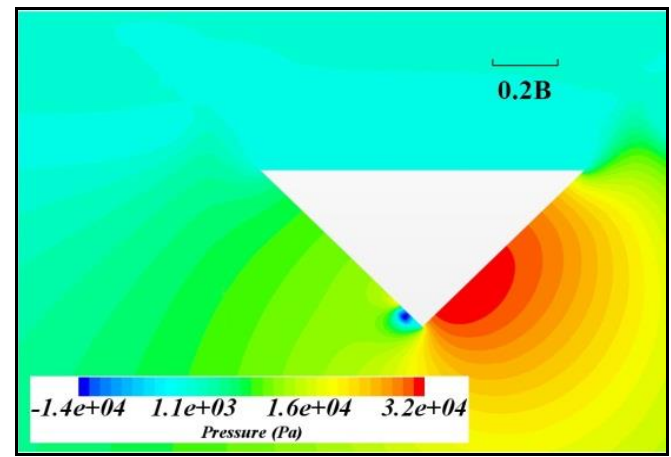

(c)

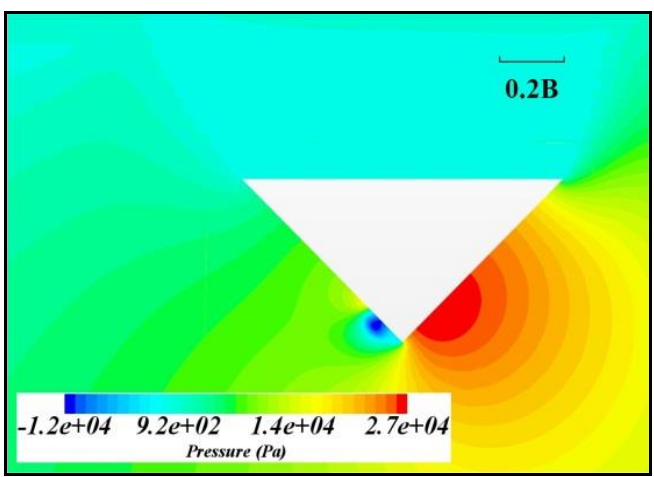

(e)

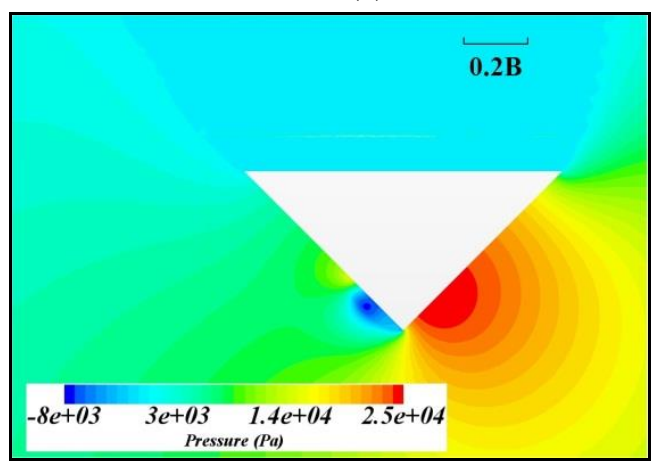

(g)

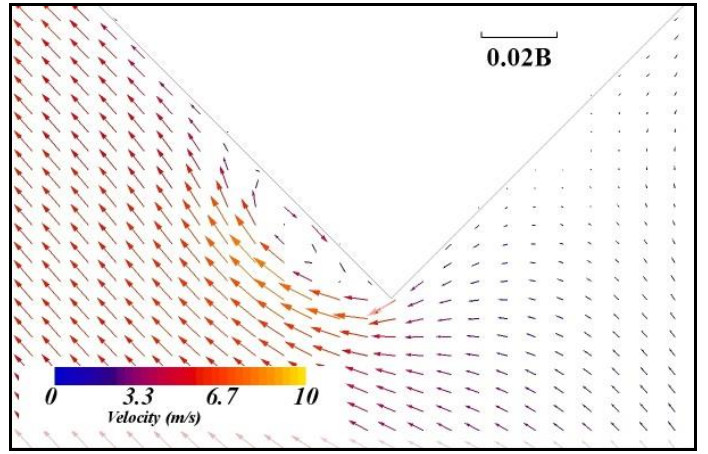

(b)

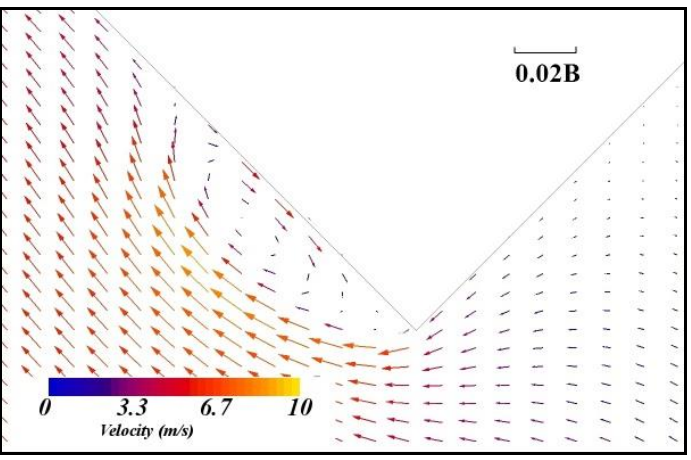

(d)

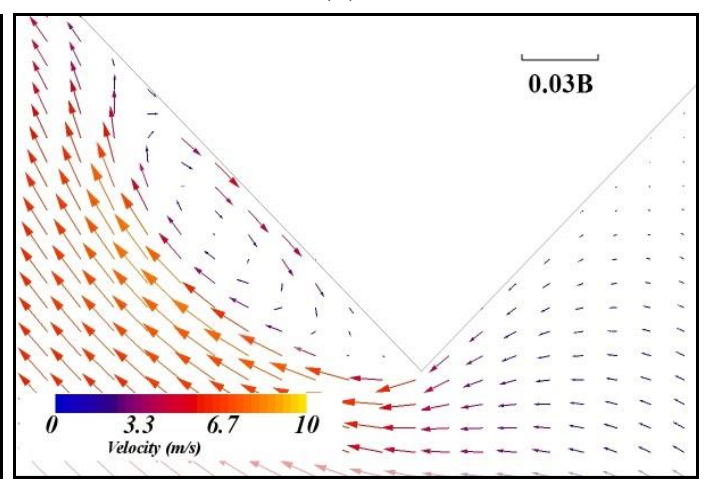

(f)

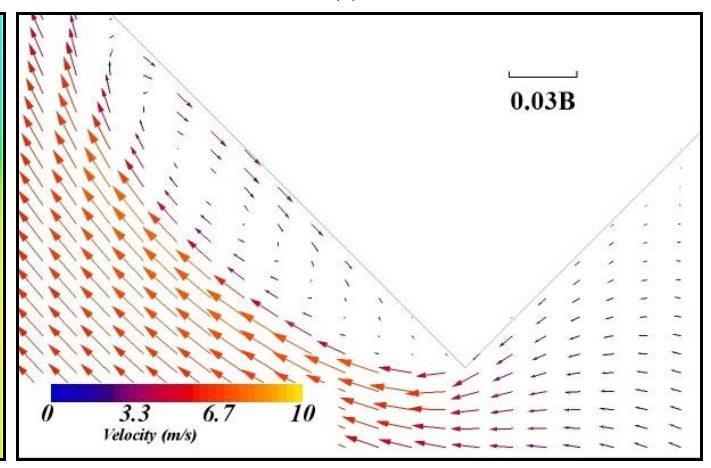

(h) 


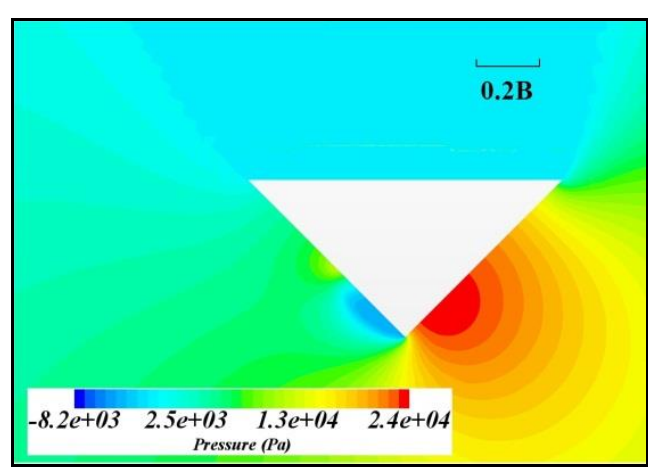

(i)

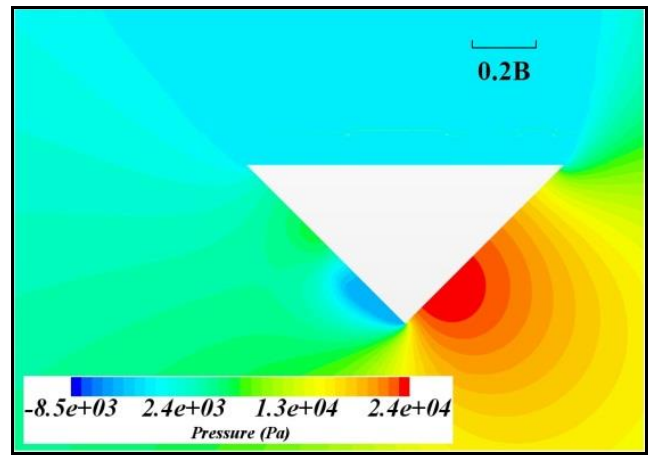

(k)

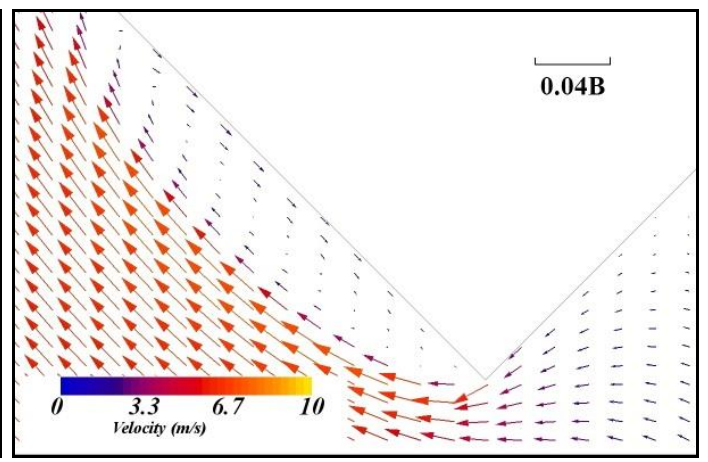

(j)

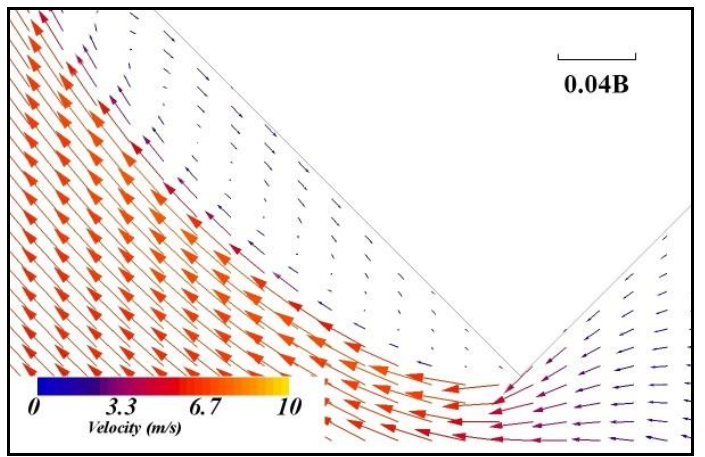

(1)

Fig.9 Wedge water entry with $\beta=45^{\circ}, \varepsilon=0.4$, the pressure contour and velocity vector at (a)(b) $V t / B=0.24$, (c)(d) $V t / B=0.48$, (e)(f) $V t / B=0.72$, (g)(h) $V t / B=0.96$, (i)(j) $V t / B=1.20$, (k)(l) $V t / B=1.68$.

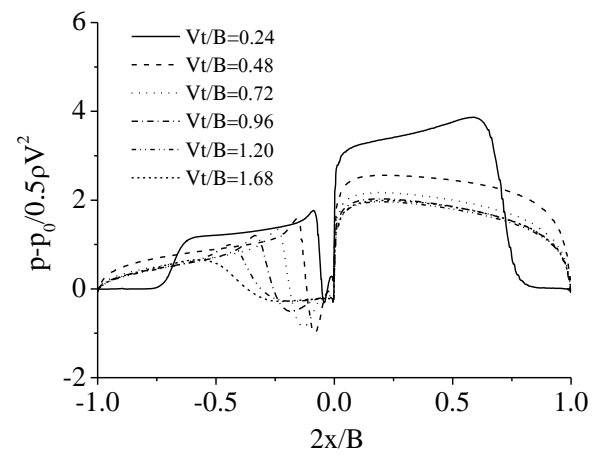

(a)

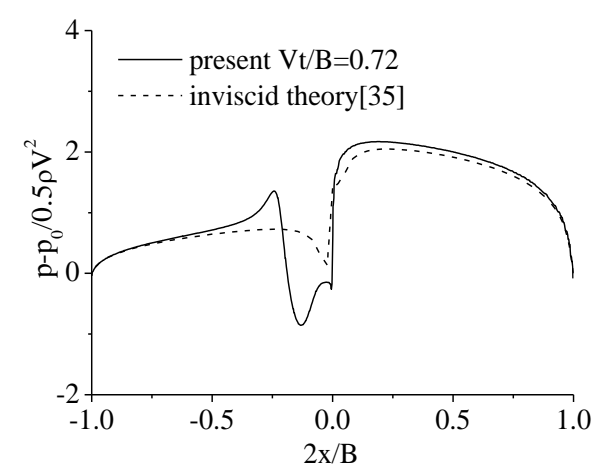

(c)

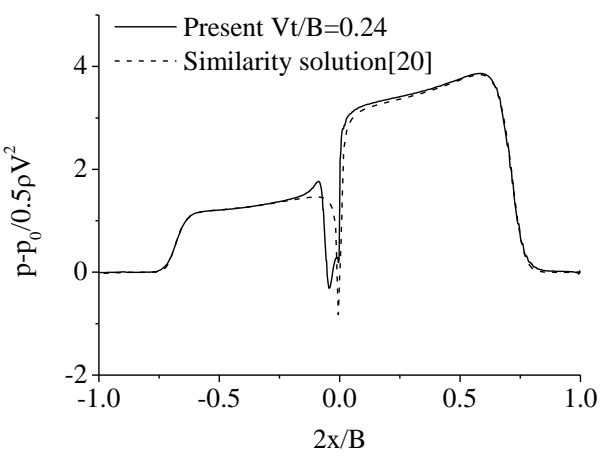

(b)

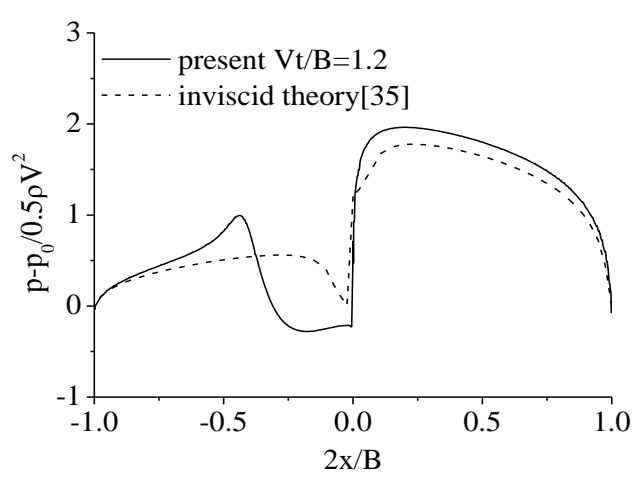

(d)

Fig.10 (a) Pressure distribution at various water entry distances with $\beta=45^{\circ}$ and $\varepsilon=0.4$, comparison with (b) the similarity solution at $V t / B=0.24$ and (c)(d) the numerical pressure with $V t / B=0.48$ and $V t / B=1.2$. 


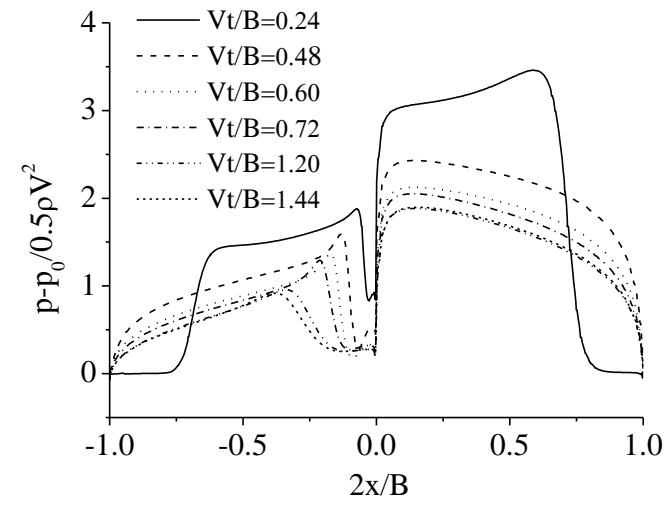

(a)

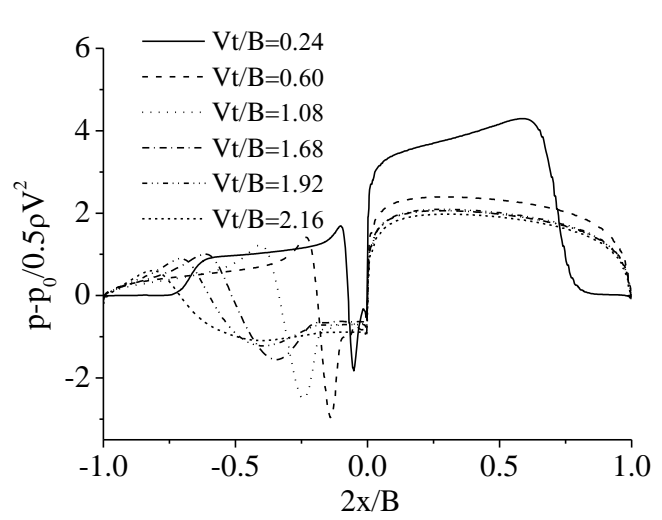

(b)

Fig.11 Pressure distribution at various water entry depth with deadrise angle $\beta=45^{\circ}$, (a) $\varepsilon=0.3$ and (b)

$$
\varepsilon=0.5
$$

Further simulations of the water entry of the wedge with deadrise angle $\beta=45^{\circ}$ at oblique velocity $\varepsilon=0.3$ and $\varepsilon=0.5$ are carried out. The pressure distributions on the wedge surface are shown in Fig.11. As the wedge entering water, the low pressure area due to vortex shedding can be found on the leeward side of the wedge. Similar with those in Fig.10, the low pressure zone expands as the wedge entering water further. We note that the lowest pressure coefficient $c_{P}$ is positive when $\varepsilon=0.3$, while the lowest pressure $c_{p}=-2.5$ when $\varepsilon=0.5$. This suggests that the cavitation and ventilation would take place if the pressure is lower than the cavitating pressure. The interactions of cavity and vortex flow would make the problem extremely complicated which is beyond the scope of present study.

The oblique water entries of wedges with $\beta=30^{\circ}, \beta=40^{\circ}, \beta=50^{\circ}, \beta=60^{\circ}$ are simulated. The entry speed $V=5 \mathrm{~m} / \mathrm{s}$ and $\varepsilon=0.4$. Fig. 12 shows the pressure distributions at various entry distances. The low pressure area is observed and expands on the leeward side as the wedge entering water. The highest pressure on the windward side decreases as $V t / B$ increases; it becomes stable as the entry distance increases further. It is interesting to see that the highest pressure coefficient on the windward side is close to 2.0, although the deadrise angles are different. We note that the negative pressure coefficient of wedges with $\beta=50^{\circ}$ and $\beta=60^{\circ}$ is considerably low; while the pressure coefficient on the leeward side of wedge with $\beta=30^{\circ}$ is positive. The typical pressure contour and the local velocity vectors at $V t / B=0.6$ are shown in Fig.13. The pressure on the windward side is much higher than that on the leeward side. The vortex shed from the wedge apex and developed into vortex bolt. We note that the induced vortex flow velocity with $\beta=60^{\circ}$ is much higher than that of wedge with $\beta=30^{\circ}$. The vortex flow is circular when $\beta=60^{\circ}$ while it is oblate when $\beta=30^{\circ}$, as shown in Fig.13. The sharper wedge apex induces stronger transverse flow and lower pressure can be found on the leeward side. The negative pressure and the development of vortex flow depend on the shape of the apex. 
X.B. Yang, G.D. Xu

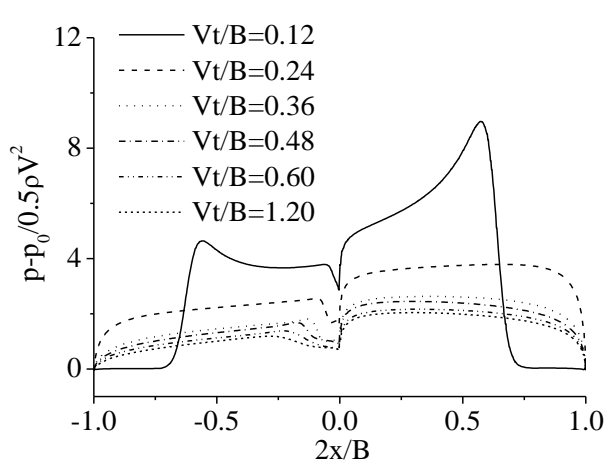

(a)

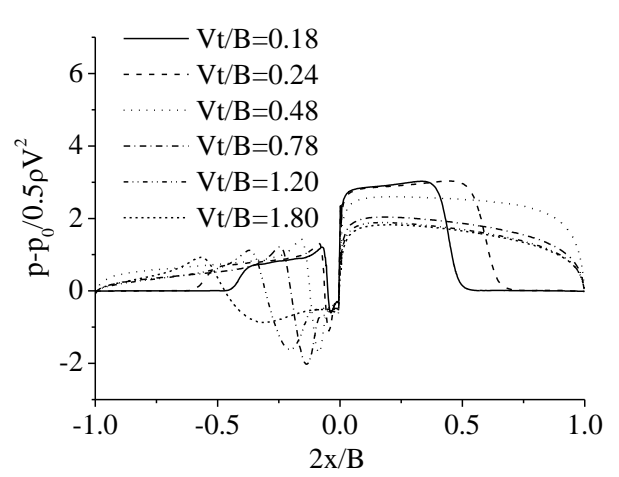

(c)
Numerical simulation of the oblique water entry of wedges with vortex shedding

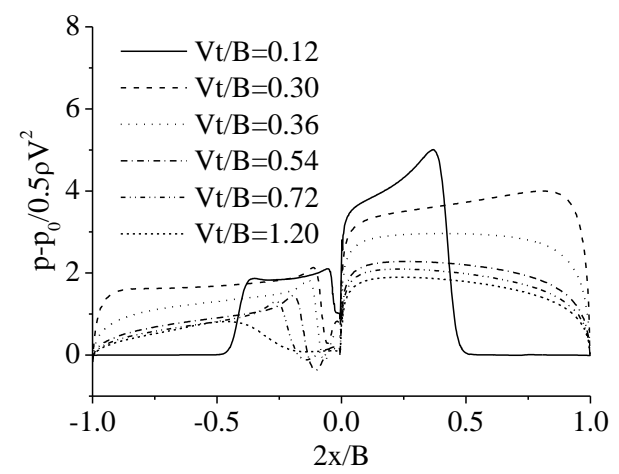

(b)

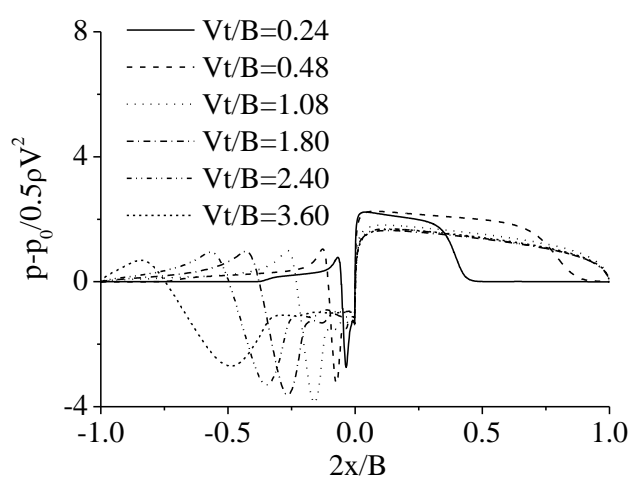

(d)

Fig.12 Pressure distribution of the wedges at various entry distance, (a) $\beta=30^{\circ}, \varepsilon=0.4$, (b) $\beta=40^{\circ}$,

$$
\varepsilon=0.4 \text {, (c) } \beta=50^{\circ}, \varepsilon=0.4 \text {, (d) } \beta=60^{\circ}, \varepsilon=0.4
$$

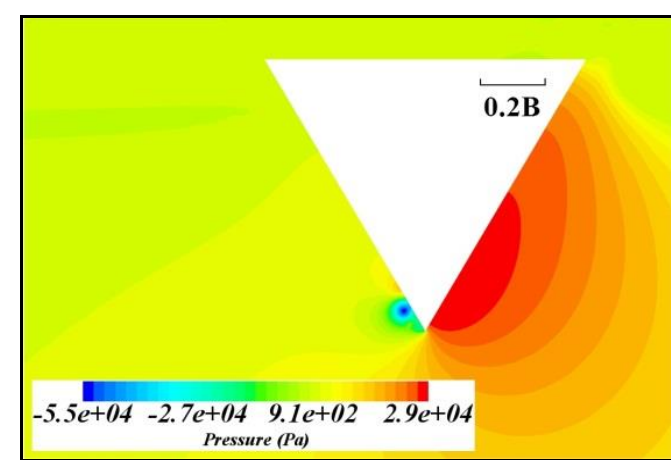

(a)

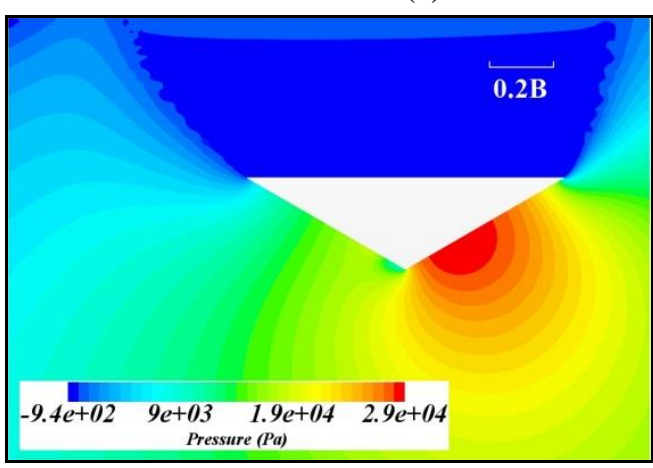

(c)

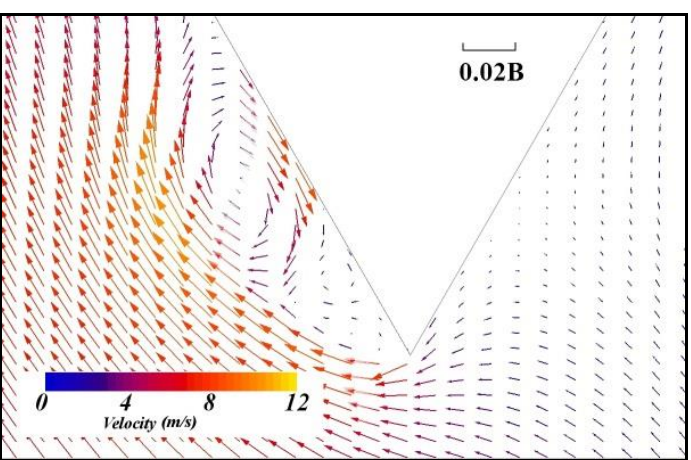

(b)

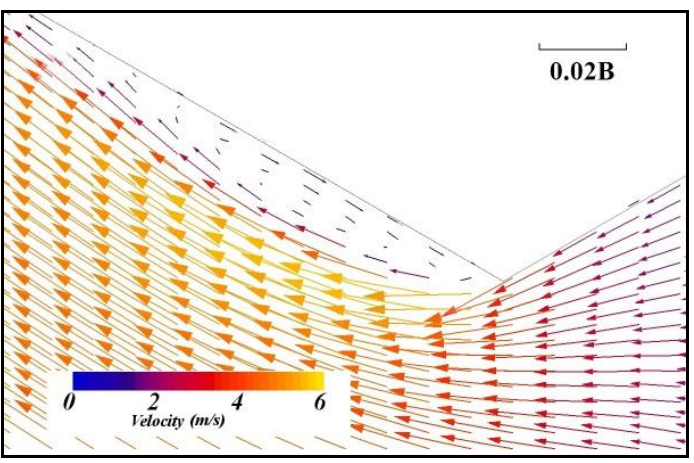

(d)

Fig.13 The pressure contour and velocity vectors of wedge water entry with $\varepsilon=0.4, V t / B=0.6$, (a)(b) $\beta=60^{\circ}$, (c)(d) $\beta=30^{\circ}$. 


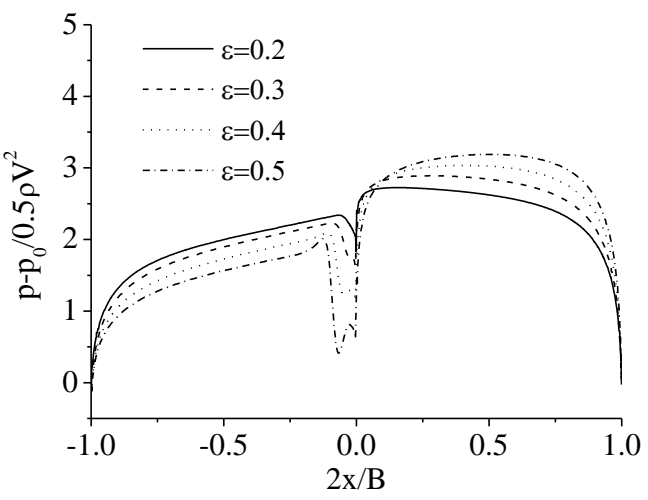

(a)

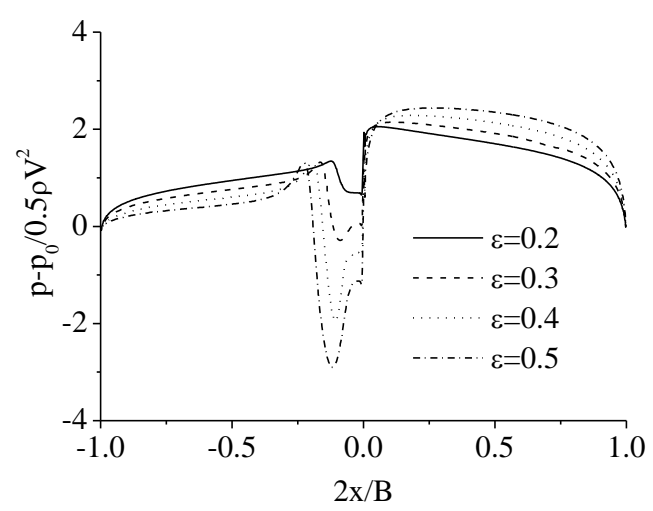

(c)

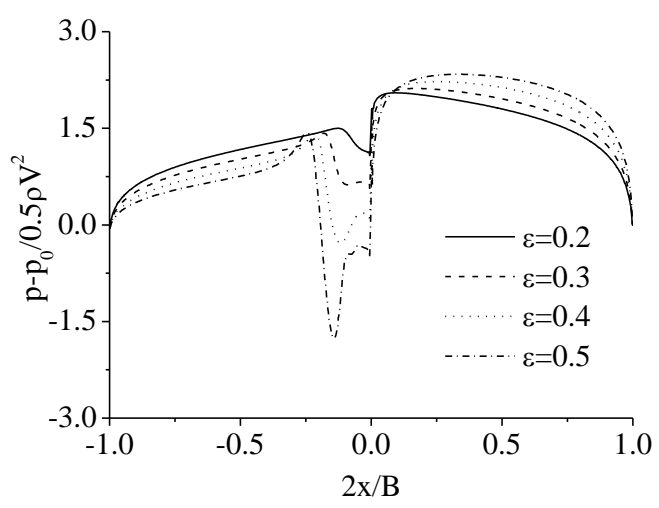

(b)

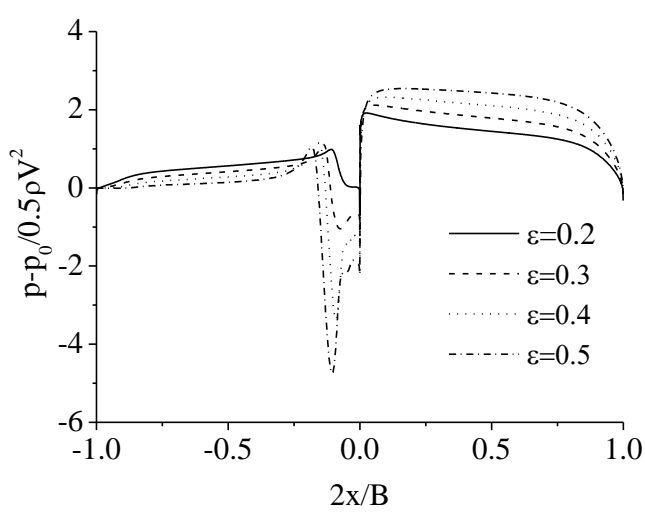

(d)

Fig.14 The typical pressure distribution with various oblique velocity, (a) $\beta=30^{\circ}, V t / B=0.30$ (b) $\beta=40^{\circ}$,

$$
V t / B=0.6 \text {, (c) } \beta=50^{\circ}, V t / B=0.6 \text { and (d) } \beta=60^{\circ}, V t / B=0.6 \text {. }
$$

The effects of oblique velocity are further investigated. The pressure distributions on the wedges surface with various $\varepsilon$ are shown in Fig.14. The positive pressure on the windward side increases while the pressure on the leeward side decreases significantly as $\varepsilon$ increases. Comparing the pressure of the wedges with $\beta=30^{\circ}, \beta=40^{\circ}, \beta=50^{\circ}$ and $\beta=60^{\circ}$, the sharper wedge apex results lower pressure on the leeward side of the wedge. The lowest pressure coefficient $c_{P}$ will decrease significantly as $\beta$ increases. The results suggest that the cavitation and ventilation will occur with larger $\varepsilon$.

\section{Conclusion}

The oblique water entries of wedges have been investigated numerically through solving the RANS equations. The overset mesh technique has been adopted in the simulations. The vortex shedding has been observed near the leeward side of wedge surface. When a sharp wedge with oblique velocity is considered, high pressure on the windward side and the low pressure area near the vortex core on the leeward side can be observed. There exists two stagnation points on the windward side and leeward side of the wedge. The highest pressure coefficient $c_{p}$ can be found at these two stagnations, one is close to the wedge apex on the windward side and the other is right above the vortex core on the leeward side. As the wedge entering water, the highest $c_{P}$ decreases on the windward side, and the low pressure area on the leeward side of the wedge expands. The numerical results show that the vortex induced flow and the lowest pressure is significantly affected by the oblique velocity and the deadrise angle. The wedge with sharp apex will induce stronger vortex flow and lower pressure, where the ventilation and separation would become an issue. 


\section{Acknowledgement}

The research is under the support of National Science Foundation of China (Grant no.51479044, 11602067) and the Fundamental Research Fund for Central Universities hosted by Harbin Engineering University (HEUCF18018). Thanks must go to the referees for their valuable suggestions and comments, upon which the quality of the work has been improved significantly.

\section{Reference}

[1] Newman, J. Marine Hydrodynamics. MIT, 1977.

[2] Von Karman T. The impact of seaplane floats during landing. Washington, DC: NACA Tech. Note 321, 1929.

[3] Wagner H. Über Stoß und Gleitvorgänge an der Oberfläche von Flüssigkeiten. Z. Angew. Math. Mech 1932;12:192-215. https://doi.org/10.1002/zamm.19320120402

[4] Armand JL, Cointe R. Hydrodynamic impact analysis of a cylinder. J. Offshore Mech. Arct. Engng 1987; 9:237-243.

[5] Korobkin AA, Puknachov VV. Initial Stage of Water Impact. Ann. Rev. Fluid Mech. 1988:159-185.

[6] Korobkin AA. Analytical models of water impact. Eur. J. Appl. Maths. 2004;15:821-838. https://doi.org/10.1017/S0956792504005765

[7] Howison SD, Ockendon JR, Oliver JM. Oblique slamming, planing and skimming. J. Engng Maths 2004; 48: 321-337. https://doi.org/10.1023/B:engi.0000018156.40420.50

[8] Mei X, Liu Y, Yue DKP. On the water impact of general two-dimensional sections. Appl. Ocean Res. 1999; 21:1-15. https://doi.org/10.1016/S0141-1187(98)00034-0

[9] Dobrovol'skaya ZN. Some problems of similarity flow of fluid with a free surface. J. Fluid Mech. 1969; 36: 805-829. https://doi.org/10.1017/S0022112069001996

[10] Zhao R, Faltinsen OM. Water entry of two-dimensional bodies. J. Fluid Mech. 1993; 246:593-612. https://doi.org/10.1017/S002211209300028X

[11] Battistin D, Iafrati A. Hydrodynamic loads during water entry of two-dimensional and axisymmetric bodies, J. Fluids and Struct. 2003;17:643-664. https://doi.org/10.1016/S0889-9746(03)00010-0

[12] Wu GX, Sun H, He YS. Numerical simulation and experimental study of water entry of a wedge in free fall motion. J. Fluids and Struct. 2004; 19:277-289. https://doi.org/10.1016/j.jfluidstructs.2004.01.001

[13] Xu GD, Duan WY, Wu GX. Simulation of water entry of a wedge through free fall in three degrees of freedom. Proc. Roy. Soc. A 2010; 466:2219-2239. https://doi.org/10.1098/rspa.2009.0614

[14] Fairlie-Clarke AC, Tveitnes T. Momentum and gravity effects during the constant velocity water entry of wedge-shaped sections. Ocean Engng 2008; 35:706-716. https://doi.org/10.1016/j.oceaneng.2006.11.011

[15] Roya Shademani, Parviz Ghadimi. Estimation of Water Entry Forces, Spray Parameters and Secondary Impact of Fixed width Wedges at Extreme Angles Using Finite Element Based Finite Volume and Volume of Fluid Methods. Brodogradnja 2016;67(1):101-124. http://dx.doi.org/10.21278/brod67101

[16] Xu L, Troesch AW, Vorus WS. Asymmetric vessel impact and planning hydrodynamics. J. Ship Res. 1998;42:187-198.

[17] Garabedian PR. Oblique water entry of a wedge. Commun. Pur. Appl. Math. 1953: 157-165. https://doi.org/10.1002/cpa.3160060201

[18] Chekin BS. The entry of a wedge into an incompressible fluid. J. Appl. Math. Mech 1989; 53:300-307. https://doi.org/10.1016/0021-8928(89)90026-9

[19] Semenov YA, Iafrati A. On the nonlinear water entry problem of asymmetric wedges. J. Fluid Mech. 2006; 547:231-256. https://doi.org/10.1017/S0022112005007329

[20] Xu GD, Duan WY, Wu GX. Numerical simulation of oblique water entry of an asymmetrical wedge. Ocean Engng 2008; 35:1597-1603. https://doi.org/10.1016/j.oceaneng.2008.08.002

[21] Judge C, Troesch AW, Perlin M. Initial water impact of a wedge at vertical and oblique angles. J. Eng. Math 2004;48:279-303.https://doi.org/10.1023/B:engi.0000018187.33001.e1

[22] Gu HB, Qian L, Causon DM, Mingham CG, Lin P. Numerical simulation of water impact of solid bodies with vertical and oblique entries. Ocean Engng 2014;75:128-137.

https://doi.org/10.1016/j.oceaneng.2013.11.021 
[23] Javanmardi N, Ghadimi P, Tavakoli S. Probing into the effects of cavitation on hydrodynamic characteristics of surface piercing propellers through numerical modelling of oblique water entry of a thin wedge. Brodogradnja. 2018: 69(2) 151-168. http://dx.doi.org/10.21278/brod69109

[24] Batchelor GK. An Introduction to Fluid Dynamics, Cambridge University Press 1967.

[25] Pullin DI. The large-scale structure of unsteady self-similar rolled-up vortex sheets. J. Fluid Mech. 1978; 88:401-430. https://doi.org/10.1017/S0022112078002189

[26] Graham JMR. The forces on sharp-edged cylinders in oscillatory flow at low Keulegan-Carpenter numbers. J. Fluid Mech. 1980; 97:331-346. https://doi.org/10.1017/S0022112080002595

[27] Pullin DI, Perry AE. Some flow visualization experiments on the starting vortex. J. Fluid Mech. 1980; 97 : 239-255. https://doi.org/10.1017/S0022112080002546

[28] Riccardi G, Iafrati A. Water impact of an asymmetric floating wedge, J. Engng. Maths. 2004;49:19-39. https://doi.org/10.1023/B:ENGI.0000014885.89822.f5

[29] Xu GD, Wu GX. Oblique water entry of a wedge with vortex shedding. Proceeding of International Workshop on Water Waves and Floating Body 2015, Bristol.

[30] Semenov YA, Wu GX. Water entry of a wedge with rolled-up vortex sheet, J. Fluid Mech. 2018. 835: 512-539. https://doi.org/10.1017/jfm.2017.766

[31] Shih TH, Liou WW, Shabbir A, Yang Z, Zhu J. A new eddy-viscosity model for high Reynolds number turbulent flows model development and validation. Comput. Fluids 1995;24(3):227-238. https://doi.org/10.1016/0045-7930(94)00032-T

[32] Hirt CW, Nichols BD. Volume of fluid (VOF) method for the dynamics of free boundaries. J. Comput Phys. 1981;39(1):201-225. https://doi.org/10.1016/0021-9991(81)90145-5

[33] Zhao R, Faltinsen OM, Aarsnes J. Water entry of arbitrary two-dimensional sections with and without separation. Proc. 21st Symposium on Naval Hydrodynamics 1996: 118-133. Trondheim, Norway.

[34] Russo S, Jalalisendi M, Falcucci G, Porfiri M. Experimental characterization of oblique and asymmetric water entry. Exp. Therm. Fluid Sci. 2018;92:141-161. https://doi.org/10.1016/j.expthermflusci.2017.10.028

[35] Bao CM, Wu GX, Xu GD. Simulation of water entry of a two-dimension finite wedge with flow detachment. J. Fluids and Struct. 2016; 65:44-59. https://doi.org/10.1016/j.jfluidstructs.2016.05.010

Submitted: $\quad$ 08.01.2018. Xiaobin Yang

Accepted: $\quad$ 15.11.2018 College of Shipbuilding Engineering, Harbin Engineering University, Harbin Accepted. 150001 China

Guodong Xu, xuguodong@hrbeu.edu.cn

College of Shipbuilding Engineering, Harbin Engineering University, Harbin 150001 China 